\title{
Tailoring the Dispersion of a Hybrid Chalcogenide/Silicon-Germanium Waveguide for Mid- Infrared Supercontinuum Generation
}

\author{
Alberto Della Torre,,${ }^{1,6}$ Milan Sinobad, ${ }^{1,2}$ Barry Luther-Davis, ${ }^{3}$ Pan Ma, ${ }^{3}$ Stephen Madden, ${ }^{3}$ Sukanta Debbarma, ${ }^{3}$ Khu \\ Vu, ${ }^{3}$ David J. Moss, ${ }^{4}$ Arnan Mitchell, ${ }^{2}$ Jean-Michel Hartmann, ${ }^{5}$ Jean-Marc Fedeli,${ }^{5}$ Christelle Monat, ${ }^{1}$ and Christian \\ Grillet $^{1}$ \\ ${ }^{1}$ Université de Lyon, Institut des Nanotechnologies de Lyon (INL), 69131 Ecully, France \\ ${ }^{2}$ School of Engineering, RMIT University, Melbourne, VIC 3001, Australia \\ ${ }^{3}$ Laser Physics Center, Australian National University, Canberra, ACT 0100, Australia \\ ${ }^{4}$ Centre for Microphotonics, Swinburne University of Technology, Hawthorn, VIC 3122, Australia \\ ${ }^{5}$ Université Grenoble Alpes, CEA-Leti, 38054 Grenoble Cedex 9, France \\ ${ }^{6}$ e-mail : alberto.della-torre@ec-lyon.fr
}

\begin{abstract}
We report mid-infrared supercontinuum generation in a silicon germanium-on-silicon waveguide. We show that the dispersion properties of the waveguide can be precisely tuned by controlling the width of a chalcogenide cladding layer.
\end{abstract}

OCIS codes: (320.6629) Supercontinuum generation; (190.4390) Nonlinear optics, Integrated optics; (140.3070) Infrared and far-infrared lasers.

\section{Introduction}

On-chip mid-infrared (mid-IR, between $3 \mu \mathrm{m}$ and $20 \mu \mathrm{m}$ ) supercontinuum (SC) generation is a technological challenge that is promising to have a strong impact in many different fields such as bio imaging, environmental sensors and security [1-5]. The prediction of great nonlinear properties, wide transparency window from 3 to $15 \mu \mathrm{m}$ and CMOS compatibility of germanium have attracted a growing interest toward germanium-based platforms [6-9]. An octave spanning supercontinuum generation up to $8.5 \mu \mathrm{m}$ has been already demonstrated by our group in a SiGe on Si waveguide [10]. The bandwidth and the coherence properties of the generated SC are mainly determined by the waveguide's dispersion profile. In general, the dispersive properties are set at the design stage and cannot be adjusted once the device has been fabricated. However, fabrication inaccuracies, surface roughness, surface contamination and the presence of defects may lead to a deviation from the targeted dispersion profile. Therefore, post-process tuning mechanisms are of great interest to post-trim the waveguide dispersion depending on the actual structure produced by fabrication. Here we demonstrate mid-IR supercontinuum generation in a highly nonlinear SiGe waveguide in both anomalous and normal dispersion. We show that it is possible to fine tune the dispersion profile a-posteriori by adding a chalcogenide cladding layer on top of the waveguide, introducing a simple postprocessing tool to control the supercontinuum dynamics and its properties.

\section{Supercontinuum Generation and dispersion tailoring}

Supercontinuum generation was achieved in a $7 \mathrm{~cm}$ long $3.75 \times 2.7 \mu \mathrm{m}^{2}$ cross-section air-clad silicon germaniumon-silicon waveguides (see fig.1 (a) for a schematic (top) and Scanning Electron Microscope image (bottom) of the waveguide). The waveguide operating in TE single mode, was pumped in anomalous dispersion (fig. 1(c), black continuous line) with $\sim 200$ fs pulses at $4 \mu \mathrm{m}$ delivered from a MIROPA-fs optical parametric amplifier with $63 \mathrm{MHz}$ repetition rate. A $1.26 \mu \mathrm{m}$ thick coating layer of chalcogenide Ge11.5As24Se64.5 was then deposited by thermal vapor deposition (see fig 1(b) for a schematic (top) and Scanning Electron Microscope image (bottom) of the waveguide). The chalcogenide clad waveguide was pumped in normal dispersion (fig 1(c), black dashed line) with pulses with the same parameters as before at $4.15 \mu \mathrm{m}$. Fig. 2 shows the experimental (blue) and simulated (red) output spectra obtained by pumping the air clad waveguide (fig. 2(a)) and the chalcogenide clad waveguide (fig. 2(b)) at $2.3 \mathrm{~kW}$ of coupled peak power. In the former case, the uneven amplitude across the spectrum (with a $-30 \mathrm{~dB}$ bandwidth of $3.55 \mu \mathrm{m}$ ) is typical of SC generation in anomalous dispersion regime. In the latter case, the narrower and smoother spectrum (with a $-30 \mathrm{~dB}$ bandwidth extending from 3.1 to $5.5 \mu \mathrm{m}$ ) is typical of SC generation in normal dispersion regime. The SC generation process was simulated by numerically solving the nonlinear Schrödinger equation using the split-step Fourier method. Nonlinearity of chalcogenide was neglected, as only $0.5 \%$ of the field in contained in the chalcogenide cladding. Experimental results are in good agreement with simulations. These results suggest that anomalous-to-normal dispersion shift occurs when the chalcogenide top cladding is added. We performed a numerical analysis to study the impact of the chalcogenide thickness on the group velocity dispersion. 
Fig. 1 (c) shows the group velocity dispersion for different thicknesses of the chalcogenide layer. As the thickness increases, the dispersion gradually decreases, (shifting toward normal when depositing $500 \mathrm{~nm}$ of chalcogenide) flattening out the dispersion profile. The dispersion profile converges as the chalcogenide thickness approaches 1 $\mu \mathrm{m}$. The possibility of controlling the dispersion profile by simply changing the thickness of the chalcogenide layer is a convenient post-processing tool to trim the group velocity dispersion depending on the actual structure produced by fabrication.

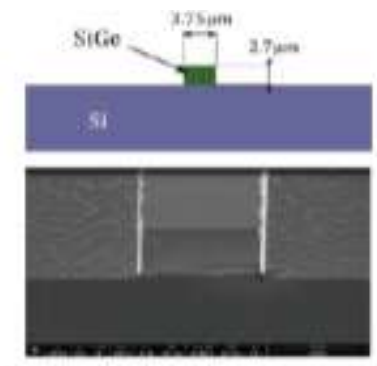

(a)

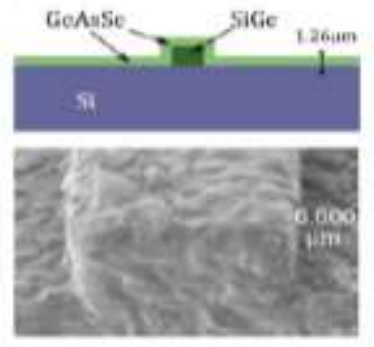

(b)

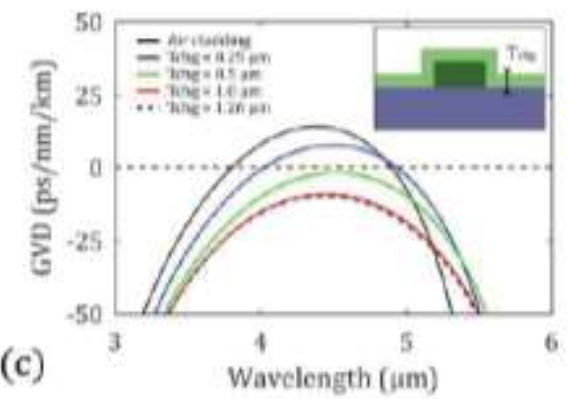

Fig. 1. Schematic (top) and Scanning Electron Microscope image (bottom) of the air clad (a) and the chalcogenide clad (b) waveguide. (c) Calculated group velocity dispersion for different thicknesses of the chalcogenide layer. The dashed black line indicates the zero dispersion. The inset show a schematic of the waveguides.
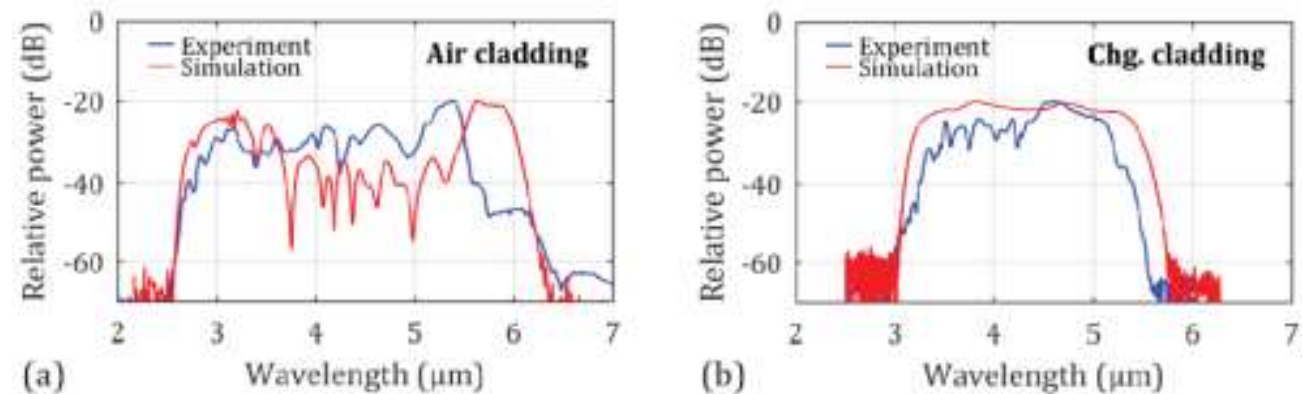

Fig. 2. Experimental (blue) and simulated (red) spectra measured out of the air cladded (a) and chalcogenide cladded (b) SiGe/Si waveguide with the same $3.75 \times 2.7 \mu \mathrm{m} 2$ core cross-section. The waveguides were pumped with pulses at $4 \mu \mathrm{m}$ and $4.15 \mu \mathrm{m}$ respectively, with $2.3 \mathrm{~kW}$ of coupled peak power.

\section{Conclusion}

In summary, we report the addition of a top chalcogenide layer as a simple post-processing technique to fine tune the dispersion profile of a nonlinear $\mathrm{SiGe}$ on $\mathrm{Si}$ waveguide for integrated SC generation. We experimentally show that, by adding a chalcogenide top layer to a ridge waveguide, anomalous-to-normal dispersion shift takes place and we numerically study the impact of the chalcogenide layer thickness on the group velocity dispersion.

Funding: Agence Nationale de la Recherche (ANR) MIRSiCOMB (ANR-17-CE24-0028), European ERC grant GRAPHICS (648546); We acknowledge the support of the LIA ALPhFA

\section{References}

[1] M. Sieger and B. Mizaikoff, "Toward On-Chip Mid-Infrared Sensors," Anal. Chem. 88, 5562-5573 (2016).

[2] L. Zhang, A. M. Agarwal, L. C. Kimerling and J. Michel, "Nonlinear Group IV photonics based on silicon and germanium: From nearinfrared to mid-infrared," Nanophotonics 3, 247-268 (2014).

[3] H. Lin et al., "Mid-infrared integrated photonics on silicon: A perspective," Nanophotonics 7, 393-420 (2017).

[4] T. Hu et al., "Silicon photonic platforms for mid-infrared applications [Invited]," Photonics Res. 5, 417 (2017).

[5] L. Wang and B. Mizaikoff, "Application of multivariate data-analysis techniques to biomedical diagnostics based on mid-infrared spectroscopy," Anal. Bioanal. Chem. 391, 1641-1654 (2008).

[6] N. K. Hon, R. Soref and B. Jalali, "The third-order nonlinear optical coefficients of Si, Ge, and $\mathrm{Si}_{1-\mathrm{x}} \mathrm{Ge}_{\mathrm{x}}$ in the midwave and longwave infrared," J. Appl. Phys. 110, 011301 (2011).

[7] G. Z. Mashanovich et al., "Germanium Mid - Infrared Photonic Devices," J. Light. Technol. 35, 624-630 (2017).

[8] M. Sinobad et al., "Broadband mid-infrared supercontinuum generation in low loss dispersion engineered silicon-germanium waveguide," in 2017 European Conference on Lasers and Electro-Optics, (Optical Society of America, Munich, Germany, 2017), paper PD_2_5.

[9] M. Sinobad et al., "Mid-wavelength Infrared Supercontinuum Generation Spanning 1.4 Octaves in a Silicon-Germanium Waveguide," in Conference on Lasers and Electro-Optics, (Optical Society of America, San Jose California, 2018), paper FTh1E.5.

[10] M. Sinobad et al., "Mid-infrared octave spanning supercontinuum generation to $8.5 \mu \mathrm{m}$ in silicon-germanium waveguides," Optica 5, 360 (2018) 